\title{
Relationship Between Angiographic Coronary Slow Flow Phenomenon and Subfoveal Choroidal Thickness: What is The Effect of Atorvastatin Therapy?
}

\section{Batur Gonenc Kanar* and Hatice Selen Kanar}

Department of Cardiology, Marmara Universitesi Tip Fakultesi, Turkey

${ }^{*}$ Corresponding author: Batur Gonenc Kanar, Department of Cardiology, Marmara Universitesi Tip Fakultesi, Turkey, Tel: +94718262236; E-mail: baturkanar@hotmail.com

Received date: March 15, 2018; Accepted date: March 26, 2018; Published date: April 06, 2018

Copyright: (C) 2018 Kanar BG, et al. This is an open-access article distributed under the terms of the Creative Commons Attribution License, which permits unrestricted use, distribution, and reproduction in any medium, provided the original author and source are credited.

Citation: Kanar BG, Kanar HS (2018) Relationship Between Angiographic Coronary Slow Flow Phenomenon and Subfoveal Choroidal Thickness: What is The Effect of Atorvastatin Therapy? Eur Exp Biol Vol. 8 No. 2:9.

\section{Abstract}

Objective: Although the pathophysiological mechanisms underlying coronary slow flow (CSF) phenomenon still remains uncertain, the microvascular dysfunction has been first implicated. The CSF phenomenon may not only affect the coronary arteries but it may also be a part of vascular problem that affect other arteries. The aim of the present study is to evaluate the relationship between CSF phenomenon and subfoveal choroidal thickness (SFCT) and to investigate the effect of short-term atorvastatin therapy on SFCT in patients with CSF.

Methods: The study population consisted of 48 patients with CSF and 41 healthy control participants. Coronary flow patterns of the patients were determined by thrombolysis in myocardial infarction (TIMI) frame count method. Spectral-domain optical coherence tomography (SD-OCT) measurements of SFCT and lipid parameters were obtained before and after 2 weeks of daily single dose of $80 \mathrm{mg}$ atorvastatin therapy.

Results: We found that patients with CSF had thinner SFCT compared with control group. There was a significant negative correlation between mean TIMI frame count and baseline SFCT $(r=-0.69, p=0.001)$. ROC curve analysis revealed that SFCT $<259 \mathrm{~m}$ predicted CSF with a $85 \%$ sensitivity and $88 \%$ specificity. The SFCT significantly increased after the short-term atorvastatin therapy.

Conclusion: There was a close negative correlation between CSF phenomenon and SFCT. Endothelial dysfunction (ED) and the resultant microvascular dysfunction might be operative on both coronary and ocular choriocapillary arteries in patients with CSF. Increase of SFCT after the short-term atorvastatin therapy might be an indicator of microvascular dysfunction improvement in patients with CSF.

Keywords: Atorvastatin; Coronary slow flow; Choroidal thickness; Spectral-domain optical coherence tomography; Microcirculation abnormality

\section{Introduction}

The coronary slow flow (CSF) phenomenon is a frequent angiographic clinical entity, characterized by delayed distal vessel opacification in the absence of significant epicardial coronary stenosis [1]. Previous histopathological studies have shown the existence of the diffused hyperplastic fibromuscular thickening of small arteries, as well as swelling and degeneration of endothelial cells with narrowing of the vascular lumina in most patients with CSF [2]. The early atherosclerosis, oxidative stress, systemic inflammatory state, and the resultant abnormalities in autacoids such as neuropeptide $Y$, endothelin-1, and thromboxane $A$ could be a pathogenetic mechanism of the CSF $[3,4]$. Based on these data, it can be suggested that an inappropriately high production of (and/or responsiveness to) these vasoconstrictors might cause the increase in resting microvascular resistance $[5,6]$. In addition, the CSF phenomenon may be a component of systemic conditions that not only affects the coronary arteries but also other arteries $[7,8]$.

The choroid is a highly fenestrated, sinusoidal vascular plexus and the site of the greatest blood flow in the body, comprising up to $85 \%$ of the blood volume in the eye to nourish the outer portion of retina. This vascular area has small arteries and veins in outer segment and wider diameters of lumens in intermediate layer and also capillary plexus in innermost layer [9]. It has been shown that many pathogenic stimuli such as diabetes and oxidative stress induce vascular dysfunction, leading to atherosclerosis, ischemia, inflammation and thrombosis may alter the regulation of retinal and choroidal blood flow [10]. According to recent studies, alteration in SFCT might be related with microvascular dysregulation entities [11-14]. OCT is a noninvasive and rapid method for multi-modal imaging the retina and choroid. In combination with an enhanced depth imaging (EDI) feature, SD-EDI OCT enables the identification of specific layers within the retina in high resolution, as well as deeper structures such as the choroid, in a way only previously possible in histological samples [15].

Previous studies indicate that some of the cholesterolindependent or "pleiotropic" effects of statins involve improving endothelial function, enhancing the stability of atherosclerotic 
plaques, decreasing oxidative stress and inflammation, and inhibiting the thrombogenic response [16]. Therefore, statins are suggested in the treatment of CSF syndrome [17-19].

The aim of the present study is to evaluate the relationship between CSF and SFCT. We also aimed to assess the possible effect of short-term atorvastatin treatment on SFCT in patients with CSF.

\section{Methods}

\section{Study design and patient population}

The study was designed to be an open-label study. Forty-six patients with angiographically proven CSF but normal epicardial coronary arteries and 43 healthy individuals were selected from patients who had undergone diagnostic coronary arteriography because of suspected coronary artery disease and were found to have normal epicardial coronary arteries without CSF. All patients and controls underwent baseline choroidal thickness evaluation by using SD-OCT. After the ophtalmological evaluation, atorvastatin $80 \mathrm{mg}$ therapy was begun to all patients with CSF. SFCT and serum lipid concentrations were performed again after two weeks of follow-up.

Patients with a history of congestive heart failure, coronary artery disease including spasm, plaque, or ectasia, valvular heart disease, hyperthyroidism, chronic obstructive pulmonary disease, ventricular preexcitation, atrioventricular conduction abnormalities and those taking medications known to alter cardiac conduction and retinochoroidal flow were excluded from the study. Patients with a history of ocular disease (glaucoma, arterial hypertension, uveitis, high myopia, age-related macular degeneration, diabetes mellitus, etc.) and/or a history of ophthalmic surgery that may have affected the choroidal vascular network were also excluded. The study was approved by local ethic committee according to the Declaration of Helsinki and the patients gave written informed consent.

\section{Ophtalmic examination and choroidal thickness evaluation}

All participants underwent a comprehensive examination including visual acuity, intraocular pressure measurement, slitlamp examination, dilated fundoscopy and SD-OCT. All SD-OCT measurements were performed during the same daily interval (10-12 am). The choroidal thickness was measured with SD-OCT (RS-3000, Nidek) manually, on the horizontal EDI line scan, in 3 separate locations: subfoveal, and $2 \mathrm{~mm}$ nasal and $2 \mathrm{~mm}$ temporal to the fovea. All choroidal thickness data were assessed by the same ophthalmologist. Measurements were performed in an area bounded by the outer limit of the retinal pigment epithelium and the inner scleral border. Mean value of both eyes were used for statistical analyzes(Figure 1). Choroidal thickness measurements were repeated by one of the ophthalmologist for a subset of images to calculate an intraobserver correlation. Intra-observer variability was 0.92 .

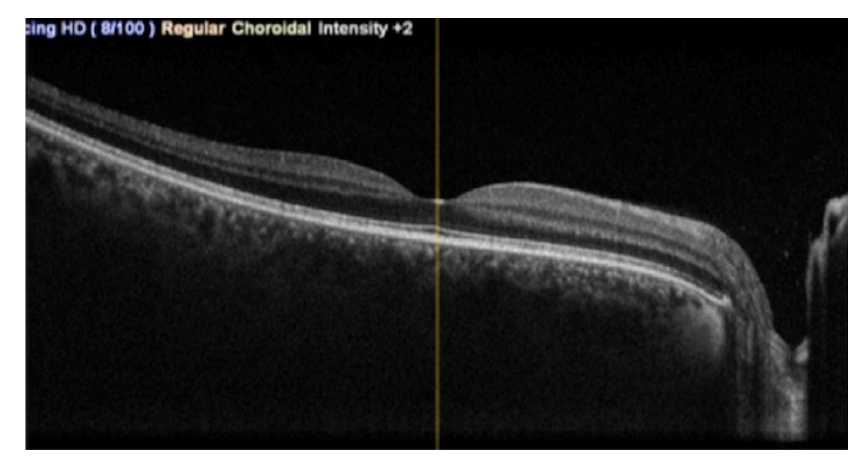

Figure 1. Spectral domain optical coherence tomography image for evaluating the subfoveal choroidal thickness.

\section{Coronary angiography and documentation of TIMI frame count}

Injection of contrast medium was carried out by an automatic injector, at a speed of 3-4 mL/s for left coronary artery and 2-3 $\mathrm{mL} / \mathrm{s}$ for right coronary arteriographies were recorded at a speed of 25 frames/s. Coronary flow was quantified objectively by an observers, who was blinded to the clinical details of the individual participants. CSF was defined according to the corrected TIMI frame count (TFC) method, and the subjects with a TFC greater than 2 standard deviations (SD) from the published normal range for the particular vessel were accepted as having CSF.18

\section{Statistical analysis}

Statistical analysis was performed using the Statistical Package for the Social Sciences (SPSS) for Windows (version 21.0; SPSS Inc., Chicago, Illinois, USA). The assumptions for linearity and homoscedasticity were tested based on the standardized residuals plots, while the assumption of normality for the dependent variable was tested using the Kolmogorov-Smirnov criterion. Continuous variables are expressed as mean value \pm standard deviation (SD). Chi- square test was employed in order to detect significant differences between categorical variables. Differences between numeric variables of two groups were tested with independent samples Student's t-test for continuous variables displaying normal distribution. Bivariate correlation analyses were done by Pearson correlation test where appropriate. The capacity of mean SFCT value in predicting presence of CSF was analyzed using Receiver Operating Characteristics (ROC) curve analysis. When a significant cut-off value was observed, the sensitivity and specificity values were presented. Statistical significance was accepted as $p$ value less than 0.05

\section{Results}

Data of 48 patients with CSF (males $=54.1 \%$, age $=51.49 .3$ years) and 41 healthy controls (males $=48.8 \%$, age $=49.78 .9$ years) were used in the analysis. CSF was observed in 3 vessels in 9 (18.7\%) patients, in 2 vessels in 23 (47.9\%) patients and in one vessel in 16 (33.3\%) patients. Left anterior descending artery 
(LAD) was affected in 38 (79.1\%) patients, circumflex artery (Cx) in 22 (45.8) patients and right coronary artery (RCA) in 29 (60.4\%) patients.

The general, biochemical, choroidal characteristics, and TIMI frame count for each major epicardial coronary artery of the subjects are presented in Table 1. TIMI frame counts of controls were significantly higher than patients with CSF compare. Mean, left, and right SFCT of patients with CSF were significantly lower than the measures of control group. There was no statistically difference between patients and control group in both clinical and biochemical data, except high sensitive $C$ reactive protein (hsCRP). There was significant negative correlation between mean TIMI frame count and SFCT ( $r$ : $-0.69, p<0.001)$ (Figure 2a). ROC curve analysis revealed that a mean SFCT $<259 \mu \mathrm{m}$ predicted CSF with $85 \%$ sensitivity and $88 \%$ specificity (area under the curve: $0.85, \mathrm{p}<0.001$, confidence interval: $0.79-0.95$ ) (Figure 2b).
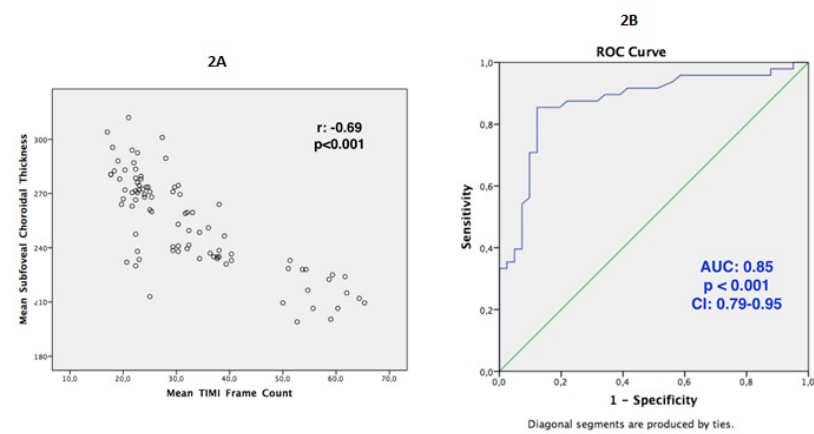

Figure 2: (a) Correlation between mean subfoveal choroidal thickness and mean TIMI frame count. (b) AUC: area under the curve, $\mathrm{Cl}$ : confidence interval.

Table 1. Demographic, angiographic, choroidal, and biochemical characteristics of the patients.

\begin{tabular}{|c|c|c|c|}
\hline & $\begin{array}{l}\text { Patients with } \\
\text { CSF (n:48) }\end{array}$ & $\begin{array}{l}\text { Control group } \\
(n: 41)\end{array}$ & $p$ value \\
\hline \multicolumn{4}{|l|}{ Clinical data } \\
\hline Age (years) & $51.4 \pm 9.3$ & $49.7 \pm 8.9$ & 0.37 \\
\hline Male/female & $26 / 22$ & $20 / 21$ & 0.61 \\
\hline $\mathrm{BMI}\left(\mathrm{kg} / \mathrm{m}^{2}\right)$ & $26.7 \pm 5.0$ & $25.0 \pm 3.1$ & 0.09 \\
\hline Systolic BP (mmHg) & $121.6 \pm 7.9$ & $122.7 \pm 8.1$ & 0.76 \\
\hline Diastolic BP (mmHg) & $77.3 \pm 4.2$ & $78.1 \pm 4.9$ & 0.68 \\
\hline Heart rate (bpm) & $74.3 \pm 8.1$ & $77.5 \pm 8.9$ & 0.89 \\
\hline Smokers & $27(56 \%)$ & $16(39 \%)$ & 0.11 \\
\hline
\end{tabular}

Table 2. Subfoveal Choroidal thickness measurements and biochemical characteristics of the patients before and after atorvastatin therapy in patients with coronary slow flow and healthy subjects.

\begin{tabular}{|c|c|c|c|c|}
\hline \multicolumn{2}{|l|}{$\mathrm{LAD}^{*}$} & $46.8 \pm 15.8$ & $21.8 \pm 3.1$ & $<0.001$ \\
\hline \multicolumn{2}{|l|}{ LCx } & $40.3 \pm 12.4$ & $21.7 \pm 3.1$ & $<0.001$ \\
\hline \multicolumn{2}{|l|}{ RCA } & $36.9 \pm 10.7$ & $22.1 \pm 2.8$ & $<0.001$ \\
\hline \multicolumn{2}{|l|}{ Mean TFC } & $41.3 \pm 12.1$ & $21.9 \pm 2.2$ & $<0.001$ \\
\hline \multicolumn{5}{|c|}{ Subfoveal choroidal thickness measurements } \\
\hline \multicolumn{2}{|l|}{$\begin{array}{l}\text { Right } \\
\text { choroidal } \\
(\mathrm{mm})\end{array}$} & $236 \pm 22$ & $300 \pm 18$ & $<0.001$ \\
\hline \multicolumn{2}{|l|}{$\begin{array}{l}\text { Left } \\
\text { choroidal } \\
(\mathrm{mm})\end{array}$} & $240 \pm 24$ & $304 \pm 19$ & $<0.001$ \\
\hline \multicolumn{2}{|l|}{$\begin{array}{l}\text { Mean } \\
\text { choroidal } \\
(\mathrm{mm})\end{array}$} & $238 \pm 23$ & $302 \pm 18$ & $<0.001$ \\
\hline \multicolumn{5}{|c|}{ Biochemical data } \\
\hline \multicolumn{2}{|c|}{$\begin{array}{l}\text { Total cholesterol } \\
(\mathrm{mg} / \mathrm{dl})\end{array}$} & $200.3 \pm 74.2$ & $189.1 \pm 70.1$ & 0.39 \\
\hline \multicolumn{2}{|c|}{$\begin{array}{l}\text { LDL- cholesterol } \\
(\mathrm{mg} / \mathrm{dl})\end{array}$} & $126.5 \pm 63.1$ & $118.3 \pm 58.6$ & 0.52 \\
\hline \multicolumn{2}{|c|}{$\begin{array}{l}\text { HDL-cholesterol } \\
(\mathrm{mg} / \mathrm{dl})\end{array}$} & $43.8 \pm 24.3$ & $43.6 \pm 24.2$ & 0.78 \\
\hline \multicolumn{2}{|c|}{ Triglyceride (mg/dl) } & $136.7 \pm 65.2$ & $135.3 \pm 64.7$ & 0.64 \\
\hline \multicolumn{2}{|c|}{ Hemoglobin (g/dl) } & $13.4 \pm 4.1$ & $13.9 \pm 3.2$ & 0.32 \\
\hline \multicolumn{2}{|c|}{ hsCRP (mg/L) } & $3.9 \pm 2.2$ & $2.2 \pm 1.5$ & 0.02 \\
\hline \multicolumn{5}{|c|}{ Bold values indicate statistical significance $p<0.05$} \\
\hline \multicolumn{5}{|c|}{$\begin{array}{l}\text { Abbreviations: BMI: body-mass index; BP: blood pressure; HDL: high-density } \\
\text { lipoprotein; hsCRP: high-sensitivity C-reactive protein; RCA: right coronary } \\
\text { artery; TFC: TIMI frame count; LAD=left anterior descending; LCx: lef } \\
\text { circumflex; LDL: Low-density lipoprotein } \\
{ }^{*} \text { Corrected TFC was given for the LAD artery. }\end{array}$} \\
\hline
\end{tabular}

SFCT measurements and biochemical characteristics before and after atorvastatin therapy are presented in Table 2. Mean SFCT increased from $238 \pm 23 \mu \mathrm{m}$ to $262 \pm 21 \mu \mathrm{m}$ after 2 weeks atorvastatin therapy $(p<0.001)$, but it was still statistically thinner than healthy controls $(262 \pm 21 \mu \mathrm{m}$ vs. $302 \pm 18 \mu \mathrm{m}$, $\mathrm{p}<0.001)$. There were no statistically significant difference after atorvastatin therapy in biochemical characteristics.

\section{Discussion}

In present study, we observed significantly lower SFCT in patients with CSF compared with controls and there was a significant negative correlation between mean TIMI frame count and SFCT before short-term atorvastatin therapy. Short-term atorvastatin therapy resulted in a significant increase in SFCT, but it was still lower than healthy control group. To the best of our knowledge our study is the first study that evaluates SFCT in patients with CSF. 


\begin{tabular}{|c|c|c|c|c|c|}
\hline & Before therapy $(n: 48)$ & $\begin{array}{l}\text { Two weeks after } \\
\text { atorvastatin therapy ( }(\mathrm{r} \text { : } \\
\text { 48) }\end{array}$ & Control group (n:41) & p value ${ }^{\alpha}$ & p value ${ }^{\beta}$ \\
\hline \multicolumn{6}{|c|}{ Choroidal thickness measurements } \\
\hline $\begin{array}{l}\text { Right choroidal thickness } \\
(\mathrm{mm})\end{array}$ & $236 \pm 22$ & $261 \pm 20$ & $300 \pm 18$ & $<0.001$ & $<0.001$ \\
\hline Left choroidal thickness (mm) & $240 \pm 24$ & $263 \pm 21$ & $304 \pm 19$ & $<0.001$ & $<0.001$ \\
\hline $\begin{array}{l}\text { Mean choroidal thickness } \\
(\mathrm{mm})\end{array}$ & $238 \pm 23$ & $262 \pm 21$ & $302 \pm 18$ & $<0.001$ & $<0.001$ \\
\hline \multicolumn{6}{|l|}{ Biochemical data } \\
\hline Total cholesterol (mg/dl) & $200.3 \pm 74.2$ & $192.4 \pm 72.4$ & $189.1 \pm 70.1$ & 0.45 & 0.73 \\
\hline LDL-cholesterol (mg/dl) & $126.5 \pm 63.1$ & $123.5 \pm 59.7$ & $118.3 \pm 58.6$ & 0.59 & 0.55 \\
\hline HDL-cholesterol (mg/dl) & $43.8 \pm 24.3$ & $44.6 \pm 25.2$ & $43.6 \pm 24.2$ & 0.12 & 0.09 \\
\hline Triglyceride (mg/dl) & $136.7 \pm 65.2$ & $122.3 \pm 59.8$ & $135.3 \pm 64.7$ & 0.08 & 0.1 \\
\hline Hemoglobin (g/dl) & $13.4 \pm 4.1$ & $13.8 \pm 4.2$ & $13.9 \pm 3.4$ & 0.24 & 0.48 \\
\hline hsCRP (mg/L) & $3.9 \pm 2.2$ & $2.9 \pm 1.8$ & $2.2 \pm 1.5$ & 0.07 & 0.02 \\
\hline \multicolumn{6}{|c|}{$\begin{array}{l}\text { Bold values indicate statistical significance } p<0.05 \text {. } \\
\text { Abbreviations: HDL: high-density lipoprotein; hsCRP: high-sensitivity C-reactive protein; LDL: Low-density lipoprotein. } \\
\text { a statistically difference between coronary slow flow patients before and after short-term atorvastatin therapy. } \\
\beta \text { statistically difference between coronary slow flow patients after short-term atorvastatin therapy and control group. }\end{array}$} \\
\hline
\end{tabular}

There have been many reports of coronary microcirculation abnormality associated with early atherosclerosis and systemic inflammatory state leading to endothelial dysfunction and increase of resting microvascular resistance in CSF phenomenon $[20,21]$. The CSF phenomenon may not only affect the coronary arteries but it may also be a part of vascular problem that affect other arteries. Sezgin et al. reported the endothelial dysfunction by using flow-mediated dilatation of the brachial artery in patients with CSF [6].

Arteriosclerotic processes and vasoconstriction resulted from oxidative stress entity and resultant increased autocoid levels, might be susceptible to choroidal microcirculation abnormality. It has been shown in animal models that arteriosclerotic changes occur in retina and choroid [22]. Recent studies related with microcirculation abnormality showed the thinning of SCFT in patients with retinitis pigmentosa [11-13] and chronic kidney disease [10] by using SD-OCT measurements. The seemingly obvious connection between the vascular components of the choroid and other vascular beds of the body has produced a number of studies on the relationship between SFCT and various cardiovascular diseases. Ahmad et al. [23] showed that patients with coronary artery diseases had a thinner macular choroid than controls and Altinkaynak et al. [24] showed that patients with congestive heart failure presented lower SFCT compared to age- and gender-matched controls. A single study evaluated the relationship between hypercholesterolemia and SFCT, showing SFCT to be significantly higher in patients with increased total cholesterol compared to controls [25]. If there were reduction in serum lipid profile, we would found decrease in SFCT values. In our study, there were no statistically significant differences between before and after serum lipid profile so increase of SCFT after statin therapy might be a reflection of generalized microvascular improvement and pleiotropic affect of short-term statin therapy.

Caliskan et al. explored the coronary flow reserve increase with atorvastatin therapy due to its anti-inflammatory effect in patients with CSF [26]. In addition, Cakmak et al. also demonstrated that simvastatin improved myocardial perfusion abnormality in patients with CSF [16]. Recently Ling et al. have shown that statin therapy improved peripheral endothelial dysfunction in CSF patients [17]. Hinoi et al. demonstrated the potent vasorelaxing effect of atorvastatin treatment on coronary microvessels in patients with normal epicardial coronary arteries [27]. We could not perform control coronary angiography to our patients with CSF after atorvastatin therapy. Hence, evaluation of the direct effect of statin therapy on coronary flow is not possible. However, increase in SFCT after statin therapy in these patients might be a reflection of improvement of microcirculation abnormality in choroidal plexus and possibly generalized endothelial function.

Improvement in endothelial functions with statin therapy might be related with anti-inflammatory effects of atorvastatin in our study which was reflected by a decrease in hsCRP levels. Li et al. showed that the plasma concentration of hSCRP increased in CSF patients compared with control group [28].

Our study has important clinical implications. Effects of medications for the treatment of patients with CSF may be assessed by measuring SCFT in clinical practice. This is especially relevant in patients without any indication for repeat coronary angiography. 


\section{Study limitation}

There are some important limitations of this study. The first limitation of this study is small sample size. Second, local ethic committee did not approve to perform control coronary angiography after statin treatment. If we had been able to perform control angiography, this would have added much more significant data to our study. Third, comparison of affect of other statins on SFCT in patients with CSF might reinforce our hypothesis. Moreover, the evaluation of short- and long-term affect of statin therapy with larger study population may improve clinical implication of our study.

\section{Conclusion}

Present study demonstrated for the first time the relationship between CSF phenomenon and SFCT by using SD-OCT. Microvascular dysregulation might be operative in both coronary and choriocapillary arteries as a systemic disorder in patients with CSF. Short-term atorvastatin theraphy was effective in the increase of SFCT, which may be an index of improvement of microvascular abnormality in patients with CSF.

\section{Author Contributions}

Dr. Batur Kanar was the primary investigator of the study. He was responsible for the design of the study, data collection, analysis of the data and drafting of the article. Dr. Hatice Selen Kanar was responsible for data collection and analysis of the data.

\section{References}

1. Mangieri EMG, Ciavolella M (1996) Slow coronary flow: clinical and histopathological features in patients with otherwise norma epicardial coronary arteries. Cathet Cardiovasc Diagn 37: 375-381.

2. Beltrame JF, Limaye SB, Wuttke RD, Horowitz JD (2003) Coronary hemodynamic and metabolic studies of the coronary slow flow phenomenon. Am Heart J 146: 84-90.

3. Beltrame JF, Limaye SB, Horowitz JD (2002) The coronary slow flow phenomenon--a new coronary microvascular disorder. Cardiology 97: 197-202

4. Karatzis EN (2005) The role of inflammatory agents in endothelial function and their contribution to atherosclerosis. Hellenic J Cardiol 46: 232-239.

5. Wang X, Nie SP (2011) The coronary slow flow phenomenon: characteristics, mechanisms and implications. Cardiovasc Diagn Ther 1: 37-43.

6. Camsari A, Pekdemir H, Ciçek D, Katircibasi T, Parmaksiz T, et al. (2004) Endothelin-1 and nitric oxide levels in patients with mitral annulus calcification. Jpn Heart J 45: 487-495.

7. Sezgin AT, Sigirci A, Barutcu I, Topal E, Sezgin N, et al. (2003) Vascular endothelial function in patients with slow coronary flow. Coron Artery Dis 14: 155-161.

8. Wang X, Geng LL, Nie SP (2010) Coronary slow flow phenomenon: a local or systemic disease? Med Hypotheses 75: 334-337.
9. Liu CH, Wang Z, Sun Y, Chen J (2017) Animal models of ocular angiogenesis: from development to pathologies. FASEB J 31: 4665-4681.

10. Hirase T, Node K (2012) Endothelial dysfunction as a cellular mechanism for vascular failure. Am J Physiol Heart Circ Physiol 302: 499-505.

11. Balmforth C, van Bragt JJ, Ruijs T, Cameron JR, Kimmitt R, et al. (2016) Chorioretinal thinning in chronic kidney disease links to inflammation and endothelial dysfunction. JCl Insight 1: 1-13.

12. Finzi A, Cellini M, Strobbe E, Campos EC (2014) ET-1 plasma levels, choroidal thickness and multifocal electroretinogram in retinitis pigmentosa. Life Sci 118: 386-390.

13. Kim H, Lee SC, Kwon KY, Lee CS (2016) Subfoveal choroidal thickness as a predictor of treatment response to anti-vascular endothelial growth factor therapy for polypoidal choroidal vasculopathy. Graefes Arch Clin Exp Ophthalmol 254: 1497-1503.

14. Sorrentino FS, Bonifazzi C, Perri P (2015) The Role of the Endothelin System in the Vascular Dysregulation Involved in Retinitis Pigmentosa. J Ophthalmol 2015: 1-6.

15. Leitgeb RA, Werkmeister RM, Blatter C, Schmetterer L (2014) Doppler optical coherence tomography. Prog Retin Eye Res 41: 26-43.

16. Liao JK, Laufs U (2005) Pleiotropic effects of statins. Annu Rev Pharmacol Toxicol 45: 89-118.

17. Cakmak M, Tanriverdi H, Cakmak N, Evrengul H (2008) Simvastatin may improve myocardial perfusion abnormality in slow coronary flow. Cardiology 110: 39-44.

18. Ling MC, Ruddy TD, de Kemp RA, Ukkonen H, Duchesne L (2005) Early effects of statin therapy on endothelial function and microvascular reactivity in patients with coronary artery disease. Am Heart J 149: 1137.

19. Gibson CM, Cannon CP, Daley WL Dodge JT Jr, Alexander B Jr, et al. (1996) TIMI frame count: a quantitative method of assessing coronary artery flow. Circulation 93: 879-888.

20. Gori T, Fineschi M (2012) Two coronary "orphan" diseases in search of clinical consideration: coronary syndromes $x$ and $y$. Cardiovasc Ther 30: 58-65.

21. Fineschi M, Bravi A, Gori T (2008) The "slow coronary flow" phenomenon: evidence of preserved coronary flow reserve despite increased resting microvascular resistances. Int J Cardiol 127: 358-361.

22. Salazar JJ, Ramírez Al, de Hoz R, Rojas B, Ruiz E, et al. Alterations in the choroid in hypercholesterolemic rabbits: reversibility after normalization of cholesterol levels. Exp Eye Res 8: 412-422.

23. Ahmad M, Kaszubski PA, Cobbs L, Reynolds H2, Smith RT (2017) Choroidal thickness in patients with coronary artery disease. PLoS One 12: 1-12.

24. Altinkaynak H, Kara N, Sayın N, Güneş H, Avşar S, et al. (2014) Subfoveal choroidal thickness in patients with chronic heart failure analyzed by spectral-domain optical coherence tomography. Curr Eye Res 39: 1123-1128.

25. Wong IY, Wong RL, Zhao P, Lai WW (2013) Choroidal thickness in relation to hypercholesterolemia on enhanced depth imaging optical coherence tomography. Retina 33: 423-428.

26. Caliskan M, Erdogan D, Gullu H, Topcu S, Ciftci O, et al. (2007) Effects of atorvastatin on coronary flow reserve in patients with slow coronary flow. Clin Cardiol 30: 475-479. 
27. Hinoi T, Matsuo S, Tadehara F, Tsujiyama S, Yamakido M (2005 Acute effect of atorvastatin on coronary circulation measured by transthoracic Doppler echocardiography in patients without coronary artery disease by angiography. Am J Cardiol 96: 89-91.
28. Li JJ, Qin XW, Li ZC, Zeng HS, Gao Z. et al. (2007) Increased plasma C-reactive protein and interleukin- 6 concentrations in patients with slow coronary flow. Clin Chim Acta 385: 43-47. 